\title{
Approach to minimizing consumption of energy in wireless sensor networks
}

\author{
Seddiki Noureddine, Benahmed Khelifa, Belgachi Mohammed \\ Department of Mathematics and Computer Science, Faculty of Exact Sciences, Tahri Mohamed University, Algeria
}

\begin{tabular}{|c|c|}
\hline Article Info & ABSTRACT \\
\hline Article history: & \multirow{10}{*}{$\begin{array}{l}\text { The Wireless Sensor Networks (WSN) technology has benefited from } \\
\text { a central position in the research space of future emerging networks by its } \\
\text { diversity of applications fields and also by its optimization techniques of its } \\
\text { various constraints, more essentially, the minimization of nodal energy } \\
\text { consumption to increase the global network lifetime. To answer this saving } \\
\text { energy problem, several solutions have been proposed at the protocol stack } \\
\text { level of the WSN. In this paper, after presenting a state of the art of this } \\
\text { technology and its conservation energy techniques at the protocol stack level, } \\
\text { we were interested in the network layer to propose a routing solution based } \\
\text { on a localization aspect that allows the creation of a virtual grid on } \\
\text { the coverage area and introduces it to the two most well-known energy } \\
\text { efficiency hierarchical routing protocols, LEACH and PEGASIS. } \\
\text { This allowed us to minimize the energy consumption and to select } \\
\text { the clusters heads in a deterministic way unlike LEACH which is done in } \\
\text { a probabilistic way and also to minimize the latency in PEGASIS, } \\
\text { by decomposing its chain into several independent chains. The simulation } \\
\text { results, under "MATLABR2015b", have shown the efficiency of our } \\
\text { approach in terms of overall residual energy and network lifetime. }\end{array}$} \\
\hline Received Apr 25, 2019 & \\
\hline Revised Nov 23, 2019 & \\
\hline Accepted Des 5, 2019 & \\
\hline Keywords: & \\
\hline CHHVG & \\
\hline CLHVG & \\
\hline Energy efficiency & \\
\hline Routing protocol & \\
\hline WSN & \\
\hline
\end{tabular}

Copyright $(2020$ Institute of Advanced Engineering and Science. All rights reserved.

\section{Corresponding Author:}

Seddiki Noureddine,

Department of Mathematics and Computer Science,

Tahri Mohamed University,

Independence Road, B.P 417, 08000, Bechar, Algeria.

Email: seddikinouredine@gmail.com

\section{INTRODUCTION}

A Wireless Sensor Network (WSN) where in English is a technique resulting from a fusion between embedded systems and wireless communications. It consists of a large number of smart, small, low-cost, power-limited sensor nodes that are able to act autonomously to collect, process and route environmental data (Temperature, wind speed, relative humidity, etc.) or real-world events (forest fires, etc.), from a monitored area to collection points called sinks. This information is then transmitted via a transport network (Internet, cellular network such as GSM, ISDN) to a processing centre where possible analyses, interpretations and decisions are made by an end-user [1].

Like any new technology, military applications were the first to benefit from modern research and development efforts in the field of WSN in the 1980s. Two important programs of the American defense agency "Defense Advanced Research Projects" (DARPA) have marked the history of the WSN, the "Distributed Sensor Networks" (DSN) program and the Senit program "Sensor Information Technology", the first visa to develop new specific techniques for sensor networks. The second, considered an ambitious program, basically sought to investigate the possibility of extending the Arpanet network (the Internet predecessor) to include sensor networks [1-2]. 
- Problematic

In recent years, there has been a growing body of interesting research on several aspects of WSN: energy, location, synchronization, mobility and topology change, quality of services, security, network processing, scalability, routing, etc. etc. But the area of research that has attracted the most interest from the research community to date, and energy conservation [3]. Each sensor node is powered by a limited-capacity on-board power source (often a battery) whose consumption for communication and computation related to information processing must be optimized, because these sensor nodes are often deployed in hostile areas that preclude any attempt at sustained maintenance, expressed as reloading or replacing their batteries in the deployment space. To deal with this problem, several solutions have been proposed [1].

One solution is to increase the capacity of the onboard battery of the sensor node but this implies an increase of its pea and its volume which is opposed to the efforts made in the field of the nano technology concerned by miniaturization and improved performance [4]. Another alternative is to extract energy "Energy Harvesting or Energy Scavenging" from the deployment environment to feed the sensor nodes directly or via charging (integrated mechanism) in real time. This energy extracted can be environmental (solar, vibration) or human (walking, body heat, breathing, blood pressure). While this solution presents an innovative and interesting idea, it has some technological limitations at present that limit its large-scale deployment [4-5].

There are other means based on power transmission for WSN, such as electromagnetic power distribution, acoustic transmitters, and light or laser. However, none of its methods are currently appropriate for WSN [6]. A fourth alternative is to design, develop and analyze (by simulation or implementation in real platforms) energy-efficient algorithms, protocols and techniques to save electrical energy within a sensor node and within the grid, whose ultimate goal is to extend the life of the WSN as long as possible while ensuring both its operationality and its performance [1]. The latter alternative falls within the field of informatics and telecommunications by its aspect related to the way in which the internal components of a sensor node consume energy as well as the way in which the nodes sensors spend energy to perform cooperative tasks, related to the nature of multi-stage ad-hoc communication, such as routing, aggregation, dissemination and change of network topology [1].

- Motivation and objective

The development of this brief is motivated by the value of this emerging WSN technology in consolidating and expanding the capabilities of the current Internet architecture to fully benefit from the services of so-called today the Internet of Things [7] which allow the possibility of communications and connections at anytime, anywhere, with anything. Also, this WSN technology occupies a central position in the research space of future emerging networks by its diversity of applications in several fields and also by its techniques of optimization of its different more essentially the increase in the lifetime of the WSN.

In addition to providing state-of-the-art information on this WSN technology and its energy minimization techniques, specifically its routing protocols, the objective of this work is to propose an energyefficient hierarchical routing approach, based on location [8-9], through the creation of a virtual grid [9-11] on the coverage area for grouping the sensor nodes in the cells of this grid. This Approach will subsequently be applied to two of the most well-known energy-efficient hierarchical routing protocols, LEACH [9] and PEGASIS [11], in order to propose two solutions derived from these in order to improve the energy saving and extend the life of the network. To simulate the two solutions based on our proposed approach, under MATLAB R2015b, we will choose as performance metrics, the network life (number of living nodes per cycle) and the remaining energy (residual) of the network. The results will be compared with the LEACH and PEGASIS protocols to assess our approach.

\section{STATE OF THE ART}

Sensor nodes are small physical devices with units that enable them to collect, process, and transmit information from the environment in which they are deployed. Among the energy-saving techniques cited in the literature [12], several solutions are based on the routing approach for routing data with energy efficiency, extending the life of the WSNs. Routing protocols can be classified through: network structure, mode of operation, communication paradigm and/or route establishment [13] as shown in Table 1.

\subsection{Low energy adaptative clustering hierarchy (leach)}

Leach [9-14] is a hierarchical protocol. The network is divided into cluster heads which act as a coordinator, and cluster members. Cluster head role rotates randomly between nodes where every node gets to be a cluster head at some point. Cluster heads are responsible of assigning the Time Division Multiple Access (TDMA) for the cluster members. When a TDMA is assigned to a certain member, it starts communicating with the cluster head while other cluster members are in their sleeping state, which decrease energy consumption. The TDMA protocol is used to regulate the channel access inside each cluster and 
decrease the interference between clusters. After the cluster heads receives data from its cluster members, the latter aggregates it and sends the aggregated data to the sink minimizing energy consumption. Although LEACH tries to reduce the number of data transmitted, the main problem in LEACH remains the direct sending of data from cluster heads to sink. However, many improvements of LEACH have been made to allow forwarding of aggregated data from cluster heads to another cluster heads all the way to the sink.

Leach is separated into two phases [15]:

- Setup Phase

- Steady State Phase

In setup phase, some nodes are elected cluster heads for the round, based on a certain formula. The elected cluster heads broadcast a massage to all other nodes informing them that they are the new cluster heads for this round. Depending on the strength of the signal that reaches it, each node decides to which cluster it wants to belong becoming cluster member to the cluster heads it chooses. Each cluster heads, after receiving message from its cluster members, creates TDMA schedule for each cluster member and broadcast it to all nodes in the cluster [16]. After the creation of clusters and TDMA schedule, steady state starts. In steady state, the sensor nodes start sensing data and forwarding it to the cluster heads in its cluster. Now the cluster heads reduce the redundancy through data aggregation and send the data to the base station. When this round ends after a certain period of time and the next round starts, all nodes return to setup phase choosing new cluster head and so on.

Table 1. Classification of routing protocols in the WSN just after the first paragraph of the section state of the art

\begin{tabular}{ll}
\hline Category & Category Representative Protocols \\
\hline Location-based Protocols & MECN, SMECN, GAF, GEAR, Span, TBF, BVGF, GeRaF \\
Data-centric Protocols & SPIN, Directed Diffusion, Rumor Routing, COUGAR, ACQUIRE, EAD, Information-Directed \\
& Routing, Gradient-Based Routing, Energy-aware Routing, Information-Directed \\
& Routing, Quorum-Based Information Dissemination, Home Agent Based Information \\
& Dissemination \\
Hierarchical Protocols & LEACH, PEGASIS, HEED, TEEN, APTEEN \\
Mobility-based Protocols & SEAD, TTDD, Joint Mobility and Routing, Data MULES, Dynamic Proxy Tree-Base Data \\
& Dissemination \\
Multipath-based Protocols & Sensor-Disjoint Multipath, Braided Multipath, N-to-1 Multipath Discovery \\
Heterogeneity-based Protocols & IDSQ, CADR, CHR \\
QoS-based protocols & SAR, SPEED, Energy-aware routing \\
\hline
\end{tabular}

\subsection{Direct diffusion (DD)}

Direct Diffusion (DD) [17] is a flat routing protocol. In direct diffusion the sink broadcasts an interest (query). Exploratory gradients are setup each time the query reaches a node, saving the path back to the sink. So once the requested data is available at a node, it is sent back to the sink through a reinforced path. We will fully explain this protocol describing its 3 stages:

- Interest propagation

- Gradient composition

- $\quad$ Data delivery

Interested propagation: the sink starts an interest message and floods it to all the nodes in the network. These messages are called exploratory message searching for data requested. After a sensor node receives this message it stores it in its cache and forwards it to its neighbour. Gradient composition: gradients are composed through different techniques. Sometimes the node with the highest energy remaining is selected. By the end of query propagation the gradient from the source back to the sink is set. We mean by source node, the node that detected the requested interest. However, many paths from source back to the sink are formed. Here the sink interferes to choose a specific path taking inconsideration several factors like link quality and enforce this path by sending the request again through it. Data delivery: after accomplishing the route between source and destination and choosing best path, now the data is transmitted to the sink.

\subsection{Geographic adaptive fidelity (GAF)}

GAF [18] is a location based routing protocol. First, the network area is divided into zones forming virtual grids. The size of these zones depends on the transmitting power and communication direction. Nodes uses GPS to associate with appoint in the grid. Different nodes that associate with the same point on the grid are equivalent regarding cost and packet routing. Nodes within each zone collaborate together dividing roles. One node is elected to stay awake to sense and report data back to the base station while others switch to sleeping mode, the more the sleeping nodes the more the energy saving. GAF can be implemented for both

Approach to minimizing consumption of energy in wireless sensor networks (Seddiki noureddine) 
mobile and non-mobile networks. However, in mobile network each node in the grid must estimate its leaving time and tell its neighbours so they can adjust their awake time according to this and keep the network active. There are 3 stages in GAF:

- Discovery: to determine neighbours in the grid.

- Active: when participating in routing.

- Sleep: when node is inactive.

\subsection{Adaptative threshold sensitive energy efficient sensor network protocol (APTEEN)}

(APTEEN) [19] Is an improvement to TEEN to overcome its shortcomings and aims at both capturing periodic data collections (LEACH) and reacting to time-critical events (TEEN). Thus, APTEEN is a hybrid clustering-based routing protocol that allows the sensor to send their sensed data periodically and react to any sudden change in the value of the sensed attribute by reporting the corresponding values to their CHs. The architecture of APTEEN is same as in TEEN, which uses the concept hierarchical clustering for energy efficient communication between source sensors and the sink. APTEEN supports three different query types namely (i) historical query, to analyse past data values, (ii) one-time query, to take a snapshot view of the network; and (iii) persistent queries, to monitor an event for a period of time. APTEEN guarantees lower energy dissipation and a larger number of sensors alive.

\subsection{Power-efficient gathering in sensor information systems (PEGASIS)}

PEGASIS [20] is an extension of the LEACH protocol, which forms chains from sensor nodes so that each node transmits and receives from a neighbour and only one node is selected from that chain to transmit to the base station (sink). The data is gathered and moves from node to node, aggregated and eventually sent to the base station. The chain construction is performed in a greedy way. Unlike LEACH, PEGASIS avoids cluster formation and uses only one node in a chain to transmit to the BS (sink) instead of using multiple nodes. A sensor transmits to its local neighbours in the data fusion phase instead of sending directly to its cluster heads as in the case of LEACH. In PEGASIS routing protocol, the construction phase assumes that all the sensors have global knowledge about the network, particularly, the positions of the sensors, and use a greedy approach. When a sensor fails or dies due to a low battery power, the chain is constructed using the same greedy approach by bypassing the failed sensor. In each round, a randomly chosen sensor node from the chain will transmit the aggregated data to the BS, thus reducing the per round energy expenditure compared to LEACH [21].

Simulation results showed that PEGASIS is able to increase the lifetime of the network twice as much the lifetime of the network under the LEACH protocol. Such performance gain is achieved through the elimination of the overhead caused by dynamic cluster formation in LEACH and through decreasing the number of transmissions and reception by using data aggregation. Although the clustering overhead is avoided, PEGASIS still requires dynamic topology adjustment since a sensor node needs to know about energy status of its neighbours in order to know where to route its data. Such topology adjustment can introduce significant overhead especially for highly utilized networks.

\subsection{Sensor protocols for information via negotiation (SPIN)}

SPIN [21] Protocol was designed to improve classic flooding protocols and overcome the problems they may cause, for example, implosion and overlap. The SPIN protocols are resource aware and resource adaptive. The sensors running the SPIN protocols are able to compute the energy consumption required to compute, send, and receive data over the network. Thus, they can make informed decisions for efficient use of their own resources. The SPIN protocols are based on two key mechanisms namely negotiation and resource adaptation. SPIN enables the sensors to negotiate with each other before any data dissemination can occur in order to avoid injecting non-useful and redundant information in the network. SPIN uses meta-data as the descriptors of the data that the sensors want to disseminate. The notion of meta-data avoids the occurrence of overlap given sensors can name the interesting portion of the data they want to get. It may be noted here that the size of the meta-data should definitely be less than that of the corresponding sensor data. Contrary to the flooding technique, each sensor is aware of its resource consumption with the help of its own resource manager that is probed by the application before any data processing or transmission. This helps the sensors to monitor and adapt to any change in their own resources.

\subsection{Speed}

SPEED [21] is another QoS routing protocol for sensor networks that provides soft real-time end-to-end guarantees. The protocol requires each node to maintain information about its neighbours and uses geographic forwarding to find the paths. In addition, SPEED [22] strives to ensure a certain speed for each packet in the network so that each application can estimate the end-to-end delay for the packets by 
dividing the distance to the sink by the speed of the packet before making the admission decision. Moreover, SPEED can provide congestion avoidance when the network is congested. The routing module in SPEED is called Stateless Geographic Non-Deterministic forwarding (SNFG) and works with four other modules at the network layer [23]. The beacon exchange mechanism collects information about the nodes and their location. Delay estimation at each node is basically made by calculating the elapsed time when an ACK is received from a neighbour as a response to a transmitted data packet. By looking at the delay values, SNGF selects the node, which meets the speed requirement. If it fails, the relay ratio of the node is checked which is calculated by looking at the miss ratios of the neighbours of a node (the nodes which could not provide the desired speed) and is fed to the SNGF module. When compared to Dynamic Source Routing (DSR) [24] and Ad-Hoc on-Demand Vector routing (AODV) [25], SPEED performs better in terms of the end-to-end delay and miss ratio. Moreover, the total transmission energy is less due to the simplicity of the routing algorithm, i.e. control packet overhead is less, and to the even traffic distribution. Such load balancing is achieved through the SNGF mechanism of dispersing packets into a large relay area [21]. SPEED does not consider any further energy metric in its routing protocol. Therefore, for more realistic understanding of SPEED's energy consumption, there is a need for comparing it to a routing protocol that is energyaware [26].

\section{COMPARISON AND SYNTHESIS}

\subsection{Comparative table}

The batch protocols that we have chosen reports of re-cent work. Our comparison was, based on comparative factors shown in the Table 2.

Table 2. Comparison between WSN routing protocols

\begin{tabular}{|c|c|c|c|c|c|}
\hline Protocol Routing & Classification & $\begin{array}{l}\text { Efficiency } \\
\text { energy }\end{array}$ & Aggregation & Scaling & $\begin{array}{c}\text { Establishment } \\
\text { road }\end{array}$ \\
\hline $\begin{array}{l}\text { SPIN : Sensor Protocols for } \\
\text { Information via Negotiation }\end{array}$ & $\begin{array}{l}\text { Flat / Center-data / } \\
\text { B-Negotiation }\end{array}$ & limited & Yes & Limited & $\begin{array}{c}\text { Reagent } \\
\text { (event) }\end{array}$ \\
\hline DD: Directed Diffusion & $\begin{array}{l}\text { Flat / Center-data / } \\
\text { B-Motion }\end{array}$ & limited & Yes & Limited & $\begin{array}{l}\text { Reagent } \\
\text { (request) }\end{array}$ \\
\hline $\begin{array}{l}\text { LEACH: Low Energy Adaptive } \\
\text { Clustering Hierarchy }\end{array}$ & $\begin{array}{l}\text { hierarchical / } \\
\text { Node-Centered }\end{array}$ & High & Yes & Bon & $\begin{array}{c}\text { Reagent } \\
\text { (Head-Cluster) }\end{array}$ \\
\hline $\begin{array}{l}\text { TEEN\&APTEEN: : [Adaptive] } \\
\text { Threshold sensitive Energy } \\
\text { Efficient sensor Network }\end{array}$ & hierarchical & High & Yes & Bon & $\begin{array}{l}\text { Active } \\
\text { Threshold } \\
\text { (Hybrid) }\end{array}$ \\
\hline $\begin{array}{l}\text { PEGASIS: The Power-Efficient } \\
\text { GAthering in Sensor Information } \\
\text { Systems }\end{array}$ & hierarchical & MAX & No & Bon & $\begin{array}{l}\text { Reagent } \\
\text { (Based- } \\
\text { Channel) }\end{array}$ \\
\hline $\begin{array}{l}\text { GAF: Geographic Adaptive } \\
\text { Fidelity }\end{array}$ & Hierarchical / Location & limited & No & Limited & Virtual Grid \\
\hline $\begin{array}{l}\text { SPEED: A real time routing } \\
\text { protocol }\end{array}$ & $\begin{array}{l}\text { Location/ } \\
\text { Centered-data / B-QoS }\end{array}$ & Low & No & Limited & Geographical \\
\hline
\end{tabular}

\subsection{Synthesis}

In the previous sections, we have established a state of the ar ton many study protocols we developed a comparative table based on a number of comparative indicators. To this end, we selected LEACH and PEGASIS protocols as the most powerful because they respond favorably to strong majority of the criteria on which we supported this study

\section{PROPOSED APPROACH}

\subsection{Approach}

In our approach to minimizing energy consumption in WSN, we introduced one of the techniques used in localization-based routing (That of the formation of a virtual grid on the coverage area) [11] to the hierarchical aspect of formation of "Clusters" groups with "Clusters heads" group leaders and chains with leaders. We applied this technique to propose two solutions derived from the most well-known hierarchical protocols in terms of energy efficiency, LEACH and PEGASIS to minimize energy consumption and extend the life of the network. For LEACH, this technique will allow the selection of group leaders in a deterministic (non-probability) manner within the cells of the virtual grid (one group leaders per cell). In addition to the use of a simple algorithm for the scheduling of transmissions which will minimize the number of messages exchanged to announce the group leaders and the joining of the nodes to the latter, and indeed allow an energy gain. 


\subsection{Assumption}

We assume for this approach:

- The base station is fixed and has an unlimited energy reserve with a significant power output (all sensor nodes are within range of the BS);

- The sensor nodes are homogeneous and stationary and have sufficient memory to avoid congestion;

- The sensor nodes have different numerical identifiers and their positions are known;

- The nodes always have data to send and this during their transmission time allocated according to a TDMA;

- The number of clusters or chains is defined.

\subsection{Clusters hierarchy on virtual grid" (CLHVG)}

This solution is based on the hierarchical energy efficiency protocol LEACH, but unlike the latter the formation of the groups is a stage that precedes the selection of the Clusters heads that are elected in a deterministic manner to its own of their groups and that rotate according to a scheduling table (TDMA) by input to their positions in the centre of the cell. The diagram in Figure 1. Shows the two stages of this solution:

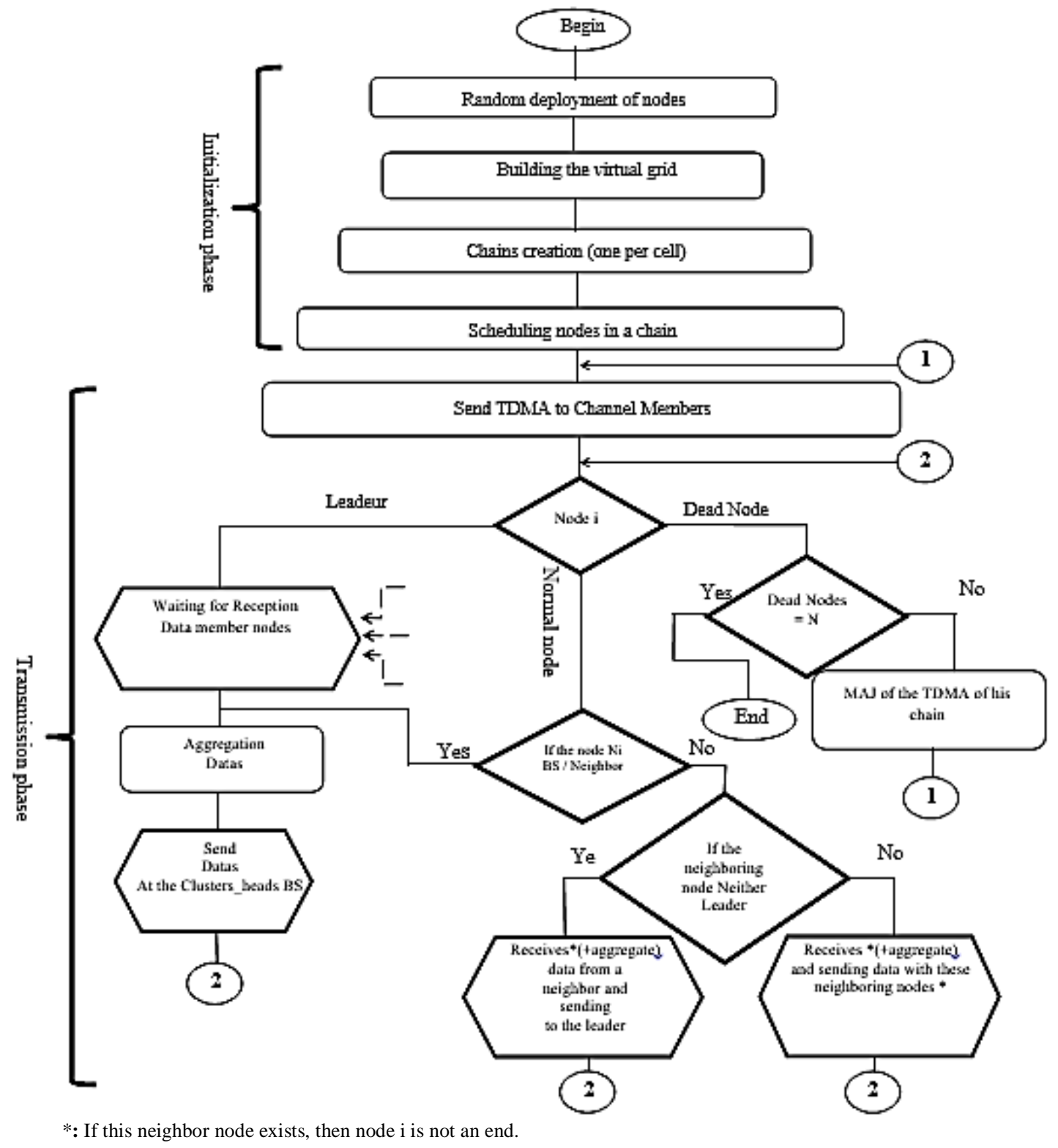

Figure 1. Organigramme CHHVG 


\subsubsection{Initialization phase}

After random deployment of the nodes, this phase allows:

- $\quad$ The construction of the virtual grid;

- $\quad$ Assignment of nodes to grid cells (cluster construction);

- The scheduling of nodes within cells by input at their distance from the centre of the cell;

\subsubsection{Initialization phase}

Once the clusters are built:

- The transmission of a scheduling table (TDMA), representing each cell to the members of the cell (cluster);

- $\quad$ The selection of the Cluster leaders is done according to their positions in this scheduling table;

- The normal nodes of each cluster transmit their data to the Clusters heads according to always the same scheduling table then the Clusters heads after aggregation transmits them in turn to the base station;

- If a node dies, it will be avoided (bayed) in the scheduling table (the cluster line will be updated and re-transmitted to members.

\subsection{Chains hierarchy on virtual grid ( CHHVG)}

The second solution is based on the hierarchical energy efficiency protocol PEGASIS, where the chain formed in this protocol will be broken down into several independent sub-chains in each of the grid cells. These sub-chains are formed by always applying the "greedy" algorithm applied in PEGASIS, but inside the cells. The leaders of these chains are elected and rotate according to a scheduling table (TDMA) by contributing to their positions. The diagram in Figure 2 shows the two stages of this solution:

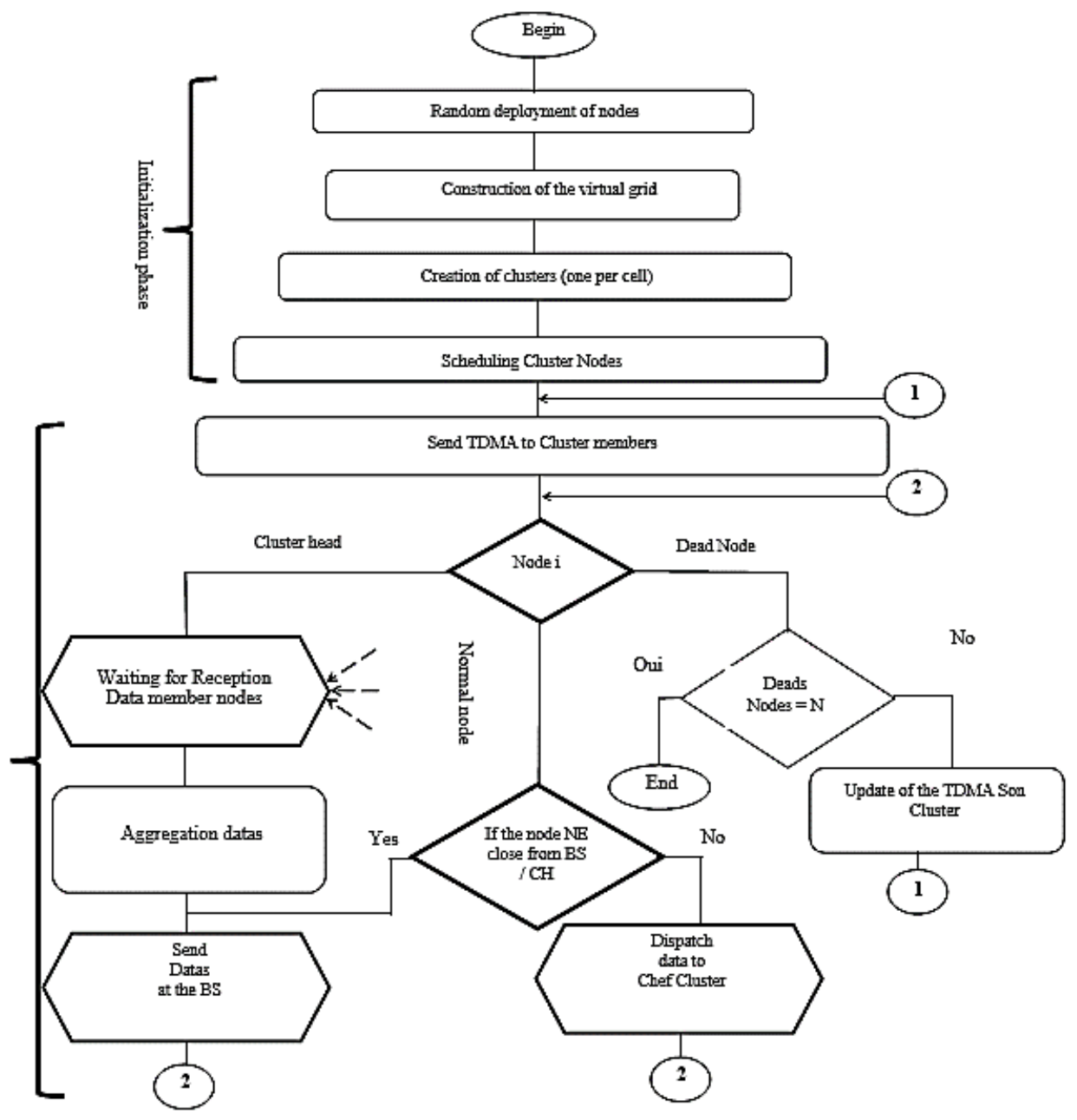

Figure 2. Organigramme CLHVG 


\subsubsection{Initialization phase}

After random deployment of the nodes, this phase allows:

- The construction of the virtual grid;

- Assignment of nodes to grid cells (chain construction);

- The Ordering of the nodes inside the cells by an algorithm "greedy" (from furthest away from the BS then the nodes closest to each other);

\subsubsection{Transmission phase}

Once the chains are built:

- The transmission of a scheduling table (TDMA), representing each cell, to the members of the cell (chain);

- The selection of Chain Heads is made according to their position in this scheduling table;

- The normal nodes of each string transmit their data to the neighboring nodes according to always the same scheduling table until the leader after aggregation transmits them in turn to the base station;

- If a node dies, it will be avoided and the scheduling table will be rebuilt and transmitted to the members of the chain;

\section{SIMULATION AND ANALYZE}

\subsection{Selection of MATLAB simulator}

The choice of MATLAB is justified by the need to use and manipulate vectors, tables and matrices (deployment of nodes, calculation of distances, and creation of scheduling tables). As well as the use of plotting and graph generation tools to evaluate the performance of the proposed solution. In addition, MATLAB is simple to study and use and thus makes it possible to evaluate energy consumption in RCSFs in a simple way. We will use the R2015b version of MATLAB.

\subsection{Simulation metrics and parameters}

\subsubsection{Simulation metrics}

- To demonstrate the effectiveness of our proposal, we have considered two metrics that will be compared in the proposed solutions with their predecessors (LEACH and PEGASIS):

- Remaining average energy: represents the amount of energy consumed by all nodes in the network.

- Network life: until all sensors exhaust their energy.

\subsection{Simulation parameters}

We established three simulation scenarios, based on the number of sensor nodes deployed and the size of the coverage area. Table 3 summarizes the values used for different simulation parameters and scenarios.

Table 3. Parameters of simulation

\begin{tabular}{|c|c|c|c|}
\hline \multirow[t]{2}{*}{ Parametres } & \multicolumn{3}{|c|}{ Values } \\
\hline & Scenario 1 & Scenario 2 & Scenario 3 \\
\hline Coverage Area & $100 \times 100 \mathrm{~m} 2$ & $100 \times 100 \mathrm{~m} 2$ & $500 \times 500 \mathrm{~m} 2$ \\
\hline $\mathrm{N}$ : Number of sensor nodes & 100 Nodes & 500 Nodes & 100 Nodes \\
\hline Position of the Base Station & $X=50, Y=50$ & $X=50, Y=50$ & $X=250, Y=250$ \\
\hline E0: Initial Energy & & 0.5 Joules & \\
\hline$E_{\text {elec }}$ : Electrical energy spent & & $50 \mathrm{~nJ} / \mathrm{bit}$ & \\
\hline$E_{a m p}$ : Dissipation energy "two - ray" & & $0.0013 \mathrm{pJ} / \mathrm{bit} / \mathrm{m} 4$ & \\
\hline $\mathrm{E}_{\mathrm{fs}}$ : Energy dissipation "Friss free space" & & $10 \mathrm{pJ} / \mathrm{bit} / \mathrm{m} 2$ & \\
\hline$E_{d a}:$ Aggregation energy & & $5 \mathrm{~nJ} / \mathrm{bit}$ & \\
\hline Package: Package Size & & 4000 bit & \\
\hline percentage of clusters & & $10 \%$ & \\
\hline
\end{tabular}

\subsection{Results and analysis}

\subsubsection{Results scenario01}

Figure 3 and Figure 4 illustrate the results of the simulation of the proposed solutions as well as those of LEACH and PEGASIS according to two metrics of performances concerning energy consumption (lifetime and energy remaining) where we can see a marked improvement in these metrics in the proposed solutions. 


\subsubsection{Results scenario02}

Figure 5 and Figure 6 show that the results of the simulation, in the case of a dense network, of the proposed solutions as well as those of LEACH and PEGASIS according to the two metrics of performances concerning the consumption of energy, are convergent. A little less for the solution "CHHVG". This can be justified by the somewhat longer life of the end nodes of the chains.

\subsubsection{Results scenario 03}

Figure 7 and Figure 8 show that the results of the simulation, in the case of a less dense network (large coverage area), according to the two performance metrics for energy consumption are significantly lower, improved for the proposed solutions than for LEACH and PEGASIS.

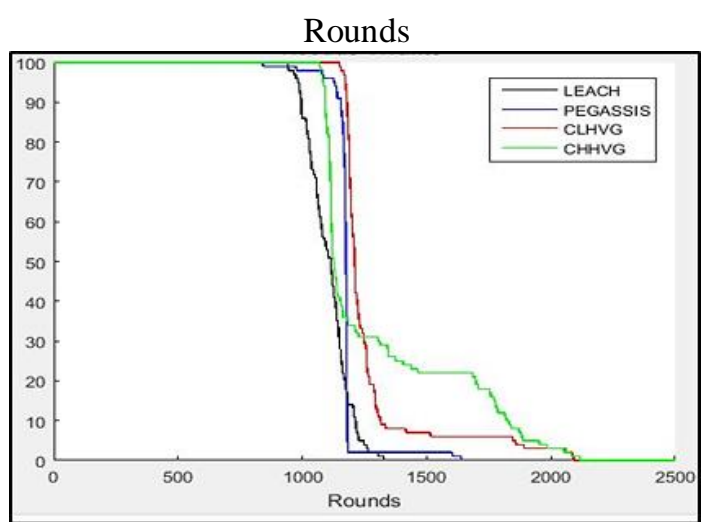

Figure 3. Lifetime

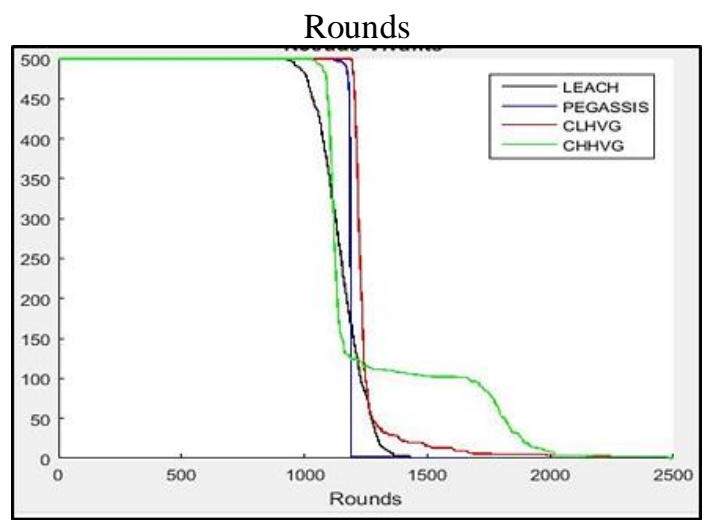

Figure 5. Lifetime

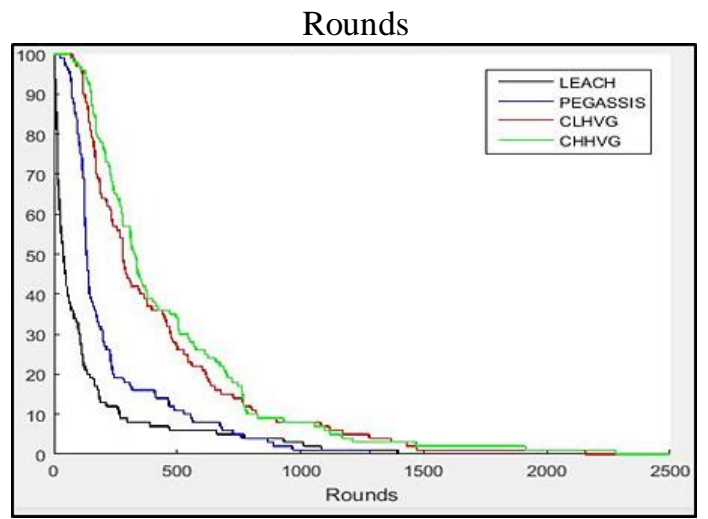

Figure 7. Lifetime

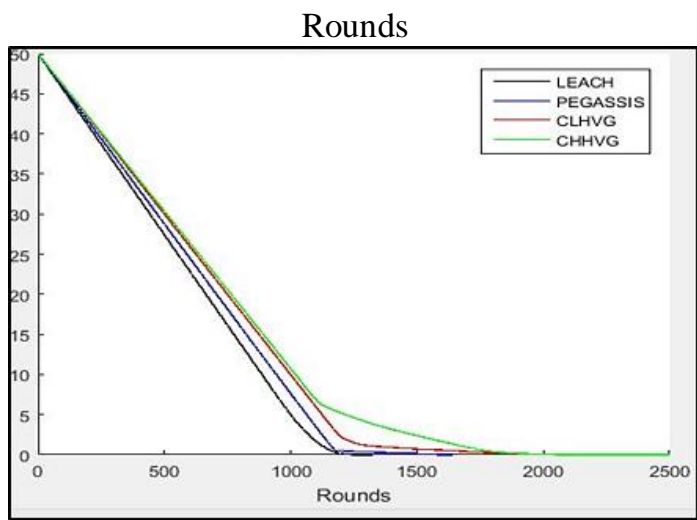

Figure 4. Remaining energy (residual)

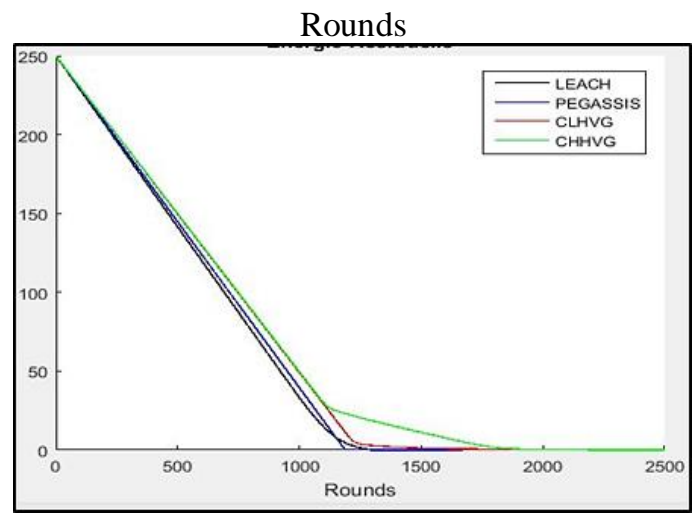

Figure 6. Remaining energy (residual)

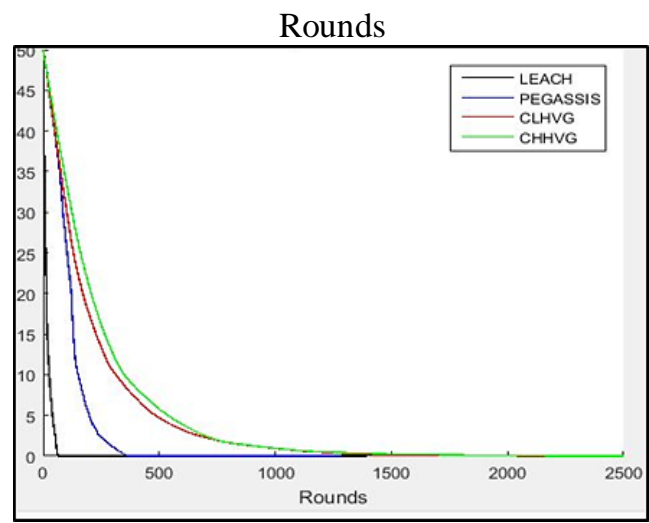

Figure 8. Remaining energy (residual)

Approach to minimizing consumption of energy in wireless sensor networks (Seddiki noureddine) 


\subsection{Synthesis}

The results recorded in the three previous scenarios show the effectiveness of our approach for minimizing the energy consumption of a wireless sensor network. However, this efficiency weakens as the density of the network or the size of the coverage area increases. This efficiency is justified by the reduction of the distances between the sensor nodes which are grouped in sub-zones thus closer to each other as well as the reduction of the number of control messages for the annunciation of the leaders of groups and the formation of these. In addition to the formation of several channels, in the solution "CHHVG" reduces the latency in the network. In the case of dense networks, the distances between neighboring sensor nodes are smaller and since the routing mechanism in the proposed solutions as well as their predecessors (LEACH and PEGASIS) are based on the distance vector this justifies the convergence of the results of the simulation obtained in the second scenario. Whereas for the less dense networks with larger coverage areas (scenario3), the distances between the sensor nodes are larger, which justifies higher energy consumption and a shorter network life?

\section{CONCLUSION AND PERSPECTIVES}

Introducing the notion of a virtual grid over the coverage area for creating groups in the WSN hierarchical routing protocols (as proposed in our approach) can further extend the network lifetime. This can even be proposed as a solution to other problems such as latency and "overhead" overhead. But this approach may present a limitation in the case of dense networks and those with large coverage area. To address the problem of network density, and as a perspective, we plan to integrate in our proposed approach, the concept of redundancy of coverage between sensor nodes member of a group (according to a defined redundancy threshold) and to allow redundant nodes to switch between the two modes sleep active alternately according to their residual energy and the number of times they were elected leader of group. This will significantly reduce the energy consumption of the sensor nodes and further extend the life of the network. For the problem of the size of the coverage area, our perspective is the integration of the notion of multi-jump routing between group leaders or chain leaders. Also, in our approach, the cells of the virtual grid are of the same form (square or rectangular) and of the same size, for another proposition, these cells can take other forms (circular, octagonal, hexagonal ... etc.) or be of different size depending on the density of the sub-areas.

\section{REFERENCES}

[1] Sanu Thomas, Thomaskutty Mathew, "Congestion bottleneck avoid routing in wireless sensor networks," International Journal of Electrical and Computer Engineering (IJECE), vol. 9, no. 6, pp. 4804-4814, Dec. 2019.

[2] C. Chong, S. P. Kumar, "Sensor Networks: Evolution, Opportunities, and Challenges," Proceedings of the IEEE, vol. 91, no. 08, pp. 1247-1256, Aug. 2003.

[3] I. Diane, "Optimization of energy consumption by taking into account measurement redundancy in sensor networks (in French)," Thèse de doctorat, Université de Toulouse, France, 2014.

[4] L. Mateu, F. Moll, "Review of Energy Harvesting Techniques and Applications for Microelectronics," Proceedings of the SPIE, the International Society for Optical Engineering, VLSI circuits and Systems II Conference, vol. 5837, pp. 359-373, 2005.

[5] L. M. Saez, "Energy Harvesting from passive human power," Electronic Engineering, PhD Thesis Project, Spain, Jan. 2004.

[6] S. Roundy, D. Steingart, L. Frechette, P. Wright, J. Rabaey, "Power Sources for Wireless Sensor Networks," Book chapter, Wireless Sensor Networks, vol. 2920, pp. 1-17, 2004.

[7] P. O. Kamgueu, "Dynamic configuration and routing for the Internet of Things," Doctoral Thesis option Networks and Telecommunications, University of Lorraine, France, 2017.

[8] J. N. Al-Karaki, A. E. Kamal., "Routing techniques in wireless sensor networks: a Survey," IEEE Wireless Communications, vol. 11, no. 6, pp. 6-28, Dec. 2004.

[9] W. R. Heinzelman, A. Chandrakasan, H. Balakrishnan., "Energy efficient communication protocol for wireless microsensor networks," In Proceedings of the 33rd Hawaii International Conference on System Sciences (HICSS'O0), vol. 2, pp. 3005-3014, 2000.

[10] P. Veeresh, R. Praveen Sam, and C. Shoba Bindu., "Reliable fault tolerance system for service composition in mobile Ad Hoc network," International Journal of Electrical and Computer Engineering (IJECE), vol. 9, no. 4, pp. 2523-2533, Aug. 2019.

[11] J. Grover, Shikha, M. Sharma, "A Study of geographic adaptive fidelity routing protocol in wireless sensor network," IOSR-JCE, vol. 16, no. 5, Ver. IV, pp. 88-96, Oct. 2014.

[12] C. Intanagonwiwat, R. Govindan, D. Estrin, "Directed diffusion: A scalable and robust communication paradigm for sensor networks," Proceedings ACM MobiCom'00, Boston, MA, pp. 56-67, Aug. 2000. 
[13] A. Manjeshwar, D. P. Agrawal, "APTEEN: A Hybrid protocol for efficient routing and comprehensive information retrieval in wireless sensor networks," in the Proceedings of the 2nd International Workshop on Parallel and Distributed Computing Issues in Wireless Networks and Mobile computing, San Francisco CA, pp. 2009-1015, Apr. 2001.

[14] L. Li, J. Y. Halpern, "Minimum energy mobile wireless networks revisited," IEEE International Conference on Communications (ICC_01), Helsinki, Finland, 2001.

[15] Y. Xu, J. Heidemann, D. Estrin, "Geography-informed energy conservation for ad-hoc routing," Proceedings ACM/IEEE MobiCom'01, Rome, Italy, pp. 70-84, Jul. 2001.

[16] S. Lindsey, C.S. Raghavendra., "PEGASIS: Power-efficient Gathering in Sensor Information System," Proceedings IEEE Aerospace Conference, Big Sky, MT, vol. 3, pp. 1125-1130, Mar. 2002.

[17] T. He, et al., "SPEED: A stateless protocol for real-time communication in sensor networks," in the Proceedings of International Conference on Distributed Computing Systems, Providence, RI, May 2003.

[18] R. Raju L, C. R. K. Reddy, "Node activity based trust and reputation estimation approach for secure and QoS routing in MANET," International Journal of Electrical and Computer Engineering (IJECE), vol. 9, no. 6, pp. 5340 5350, Dec. 2019.

[19] Freebersyser, J. A. Leiner, "A DoD perspective on mobile ad hoc networks," In:Perkins, C. (Ed.) Ad Hoc Networking, Addison Wesley, Reading, MA, pp. 29, 2001.

[20] S.Chauhan, G. Kaur, "A Virtual Grid-Based Dynamic RoutesAdjustment (VGDRA) Scheme for Wireless Sensor Networks Based on Sink Mobility," In International Research Journal of Engineering and Technology (IRJET), vol. 04, pp. 212-216, Jan. 2017.

[21] H. Ouldzira, H. Lagraini, A. Mouhsen, M. Chhiba, A. Tabyaoui., "MG-leach: an enhanced leach protocol for wireless sensor network," International Journal of Electrical and Computer Engineering (IJECE), vol. 9, no. 4, pp. 3139-3145, Aug. 2019.

[22] D. Imededdin, et al, "Design and implementation of low power consumption wireless sensor node," TELKOMNIKA (Telecommunication, Computing, Electronics and Control), vol. 17, no. 6, pp. 2729-2734, Dec. 2019.

[23] M. Thangaraj, P.P. Ponmalar, "Swarm Intelligence based Secured Data Aggregation in Wireless Sensor Networks," 2014 IEEE International Conference on Computational Intelligence and Computing Research, 2014.

[24] B. Prathiba, K. Jaya Sankar, V. Sumalatha, "Novel framework of retaining maximum data quality and energy efficiency in reconfigurable wireless sensor network," International Journal of Electrical and Computer Engineering (IJECE), vol. 9, no. 4, pp. 2893-2901, Aug. 2019.

[25] Karuna Babber, Rajneesh Randhva, "Energy Efficient Clustering with Secured Data Transmission Technique for Wireless Sensor Networks," 2016 International Conference on Computing for Sustainable Global Development (INDIACom), 2016.

[26] Ajay. K. Talele, Suraj G. Patil and Nilkanth B. Chopade, "A Survey on Data Routing and AggregationTechniques for Wireless SensorNetworks," 2015 International Conference on Pervasive Computing (ICPC), 2016.

\section{BIOGRAPHIES OF AUTHORS}

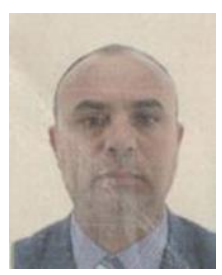

Dr. Seddiki Noureddine, assitant researcher at the University of Tahri Mohamed Bechar Algeria, Head of Department of Mathematics and Computer Science, Faculty of Sciences Exact. Member of the research team " Routing with real-time QoS in the WSN, University of Tahri Mohamed Bechar Algeria.

Area of expertise

- Wireless sensor network

- Routing protocol

- Internet of things (IoT)

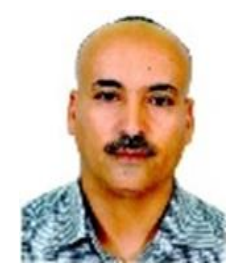

Khelifa, has done his research for Ph.D. degree in the field of sensor networks wireless at the school of computing and mathematical sciences at Liverpool john moores university, UK. He has 26 years of experience in education and research at the University Tahri Mohamed Bechar, Algeria. Khelifa has also several international publications in the fields: wireless sensor networks, security, surveillance, smart grid, Internet of things, and smart irrigation. Khelifa holds Engineer, Magister degrees and PhD in Computer sciences in University of Oran Algeria.

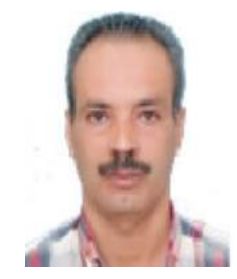

Belghachi Mohammed, assitant researcher at the University of Tahri Mohamed Bechar Algeria, Head of Department of Mathematics and Computer Science, Faculty of Sciences Exact. Member of the research team "Networks Ad-hoc" of the laboratory STIC (Information Systems and Technologies and Communication), University Abou-Bekr Belkaid Tlemcen Algeria.

Area of expertise

- Wireless sensor network

- Routing protocol

- Internet of things (IoT)

- Internet of vehicles (IoT)

- Routing security 